Pesq. Vet. Bras. 31(2):151-157, fevereiro 2011

\title{
Combinação de drogas antiparasitárias como uma alternativa para o controle de nematódeos gastrintestinais multirresistentes em ovinos ${ }^{1}$
}

\author{
Alfredo Skrebsky Cezar ${ }^{2 *}$, Henrique Olmedo Ribas ${ }^{3}$, Felipe Lamberti Pivoto ${ }^{4}$, \\ Luís Antônio Sangioni ${ }^{5}$ e Fernanda Silveira Flôres Vogel ${ }^{5}$
}

\begin{abstract}
Cezar A.S., Ribas H.O., Pivoto F.L., Sangioni L.A. \& Vogel F.S.F. 2011. [Combination of drugs as an alternative to control multi-resistant gastrointestinal nematodes in sheep.] Combinação de drogas antiparasitárias como uma alternativa para o controle de nematódeos gastrintestinais multirresistentes em ovinos. Pesquisa Veterinária Brasileira 31(2):151-157. Programa de Pós-Graduação em Medicina Veterinária, Universidade Federal de Santa Maria, Av. Roraima 1000, Santa Maria, RS 97105-900, Brazil. E-mail: alfredosps@ @otmail.com

This study was conducted to find effective anthelmintic treatments on a multi-resistant population of gastrointestinal nematodes of sheep, which was found resisting to all the classes of nematicidal drugs available in the Brazilian market. We took as a starting point the data obtained in a previous study performed in the targeted flock, in which were tested conventional treatments. Thus, using the calculated efficacy of the drugs (tested previously) for the genera of nematodes found in the flock, we selected drugs which, combined or used in higher doses, could improve the efficacy of the treatments. We evaluated the anthelmintic action of each treatment using the percentage of reduction of eggs in the feces, along with larvae cultures. A combination of moxidectin $1 \%+$ disophenol $20 \%$ showed $99 \%$ of efficacy, and combinations of moxidectin $1 \%+$ trichlorphon $10 \%$ and moxidectin $1 \%$ + levamisole phosphate $22.3 \%$ showed efficacy over $90 \%$. Combinations of drugs, with different mechanisms of action, can provide effective anthelmintic treatments on multidrug-resistant populations of gastrointestinal nematodes of sheep.
\end{abstract}

INDEX TERMS: Multidrug resistance, anthelmintics, association, efficacy, ruminants.

RESUMO.- Este estudo foi realizado com o objetivo de encontrarem-se tratamentos anti-helmínticos eficazes sobre uma população de nematódeos gastrintestinais de ovinos, a qual desenvolveu resistência a todas as classes de drogas com ação nematicida disponíveis para ruminantes no mercado brasileiro. Valendo-se da eficácia calculada de tratamentos convencionais a partir de um estudo prévio e, as-

\footnotetext{
${ }^{1}$ Recebido em 5 de maio de 2010.

Aceito para publicação em 18 de setembro de 2010.

2 Programa de Pós-Graduação em Medicina Veterinária (PPGMV), Universidade Federal de Santa Maria (UFSM), Santa Maria, RS 97105900, Brasil. *Autor para correspondência: alfredosps@ @otmail.com

${ }^{3}$ Médico Veterinário autônomo. Endereço particular: Rua Major Duarte 351, Apto 401, Santa Maria, RS 97050460.

${ }^{4}$ Graduando em Medicina Veterinária, UFSM, Santa Maria, RS.

${ }^{5}$ Departamento de Medicina Veterinária Preventiva (DMVP), Centro de Ciências Rurais (CCR), UFSM, Santa Maria, RS.
}

sim, conhecendo-se sua ação sobre diferentes gêneros de nematódeos presentes no rebanho, selecionaram-se drogas as quais, utilizadas em combinações ou em altas doses, pudessem aumentar significativamente a eficácia dos tratamentos. Utilizou-se o percentual de redução da contagem de ovos nas fezes e as culturas de larvas para avaliar a ação anti-helmíntica dos tratamentos testados. A combinação de moxidectina $1 \%$ + disofenol $20 \%$ apresentou $99 \%$ de eficácia, e as combinações de moxidectina $1 \%+$ triclorfon $10 \%$ e de moxidectina $1 \%$ + fosfato de levamisol $22,3 \%$ superaram os $90 \%$ de eficácia. A utilização de combinações de princípios ativos, com diferentes mecanismos de ação, pode promover a recuperação da eficácia dos tratamentos anti-helmínticos sobre populações multirresistentes de nematódeos gastrintestinais de ovinos.

TERMOS DE INDEXAÇÃO: Resistência múltipla, anti-helmínticos, associação, eficácia, ruminantes. 


\section{INTRODUÇÃO}

A resistência múltipla de nematódeos gastrintestinais de pequenos ruminantes às três principais classes de drogas com ação em endoparasitas (avermectinas/milbemicinas, benzimidazóis e imidazotiazóis) tem se tornado um problema comum ao redor do mundo (Mwamachi et al. 1995, Sargison et al. 2007, Traversa et al. 2007). Por sua vez, o desenvolvimento de novas drogas antiparasitárias é dificultado por uma série de fatores de ordem técnica e econômica (McKellar \& Jackson 2004, Hopkins et al. 2007, Woods \& Williams 2007) e, com isso, torna-se necessário recorrer a outras classes menos consagradas de anti-helmínticos (como nitrofenóis, salicilanilidas e organofosfatos) (McKellar \& Jackson 2004) para que se obtenham tratamentos eficazes nesses rebanhos. Mas, o que fazer quando o manejo antiparasitário equivocado resulta em resistência múltipla a todas as classes de drogas com ação nematicida disponíveis no mercado?

Os métodos de controle alternativo de endoparasitas, com base no manejo dos animais e das pastagens, no estímulo à resposta imune do hospedeiro, no controle biológico e na fitoterapia, têm se mostrado promissores, e são importantes para a sustentabilidade do controle de parasitas nos rebanhos. Porém, ainda devem ser vistos como complementares a tratamentos antiparasitários convencionais e eficazes (Waller \& Thamsborg 2004, van Wyk et al. 2006). Por conta disso, é necessário que se encontrem alternativas para a recuperação da eficácia das drogas antiparasitárias sobre populações de helmintos multirresistentes.

Como algumas dessas alternativas podem-se testar: fármacos de alta concentração (longa ação), aumento das doses de medicamentos, aplicações de um mesmo fármaco em dias consecutivos, e combinações comerciais de princípios ativos. Porém, alguns pontos negativos em relação a essas práticas precisam ser considerados, tais como: a ineficácia de drogas de longa ação em cepas resistentes ao(s) seu(s) princípio(s) ativo(s), além da maior pressão de seleção provocada por essas drogas (Barnes et al. 1995, Dobson et al. 1996, Yazwinski et al. 2006, Cezar et al. 2010a); a pressão de seleção (Smith et al. 1999) e os efeitos adversos (Bakima et al. 1989, Borges et al. 1999) mais intensos, que podem resultar de superdosagens; o manejo excessivo dos animais, e os altos custos inerentes a aplicação de uma ou mais drogas em dias consecutivos; e uma gama mais restrita de opções de combinações de princípios ativos, no caso de se utilizarem formulações (combinadas) comerciais.

As combinações de drogas têm sido empregadas para o controle de parasitas resistentes ou para o aumento da eficácia de tratamentos antiparasitários em humanos (Albonico et al. 2003, Pink et al. 2005, Hu et al. 2010) e, particularmente, para o controle de ectoparasitas resistentes em bovinos (George et al. 2004, Furlong et al. 2007, Martins et al. 2008). Embora essa estratégia tenha sido, proporcionalmente, pouco explorada para endoparasitas, o aparecimento de resistência parasitária múltipla em rebanhos de pequenos ruminantes instigou alguns estudos nesse sentido (Anderson et al. 1988, Miller \& Craig 1996, Entrocasso et al. 2008). Ao instituírem-se tratamentos com combinações de drogas é possível manter as dosagens recomendadas para cada fármaco e combinar bases pertencentes a grupos químicos distintos, com diferentes mecanismos de ação. Essas diferenças dificultam a adaptação dos genótipos parasitários aos tratamentos (Barnes et al. 1995, Geerts \& Gryseels 2000, Hu et al. 2010).

No presente estudo, índices de eficácia previamente conhecidos de anti-helmínticos e endectocidas testados no rebanho-alvo (Cezar et al. 2010b), foram empregados como ferramentas para a identificação de drogas candidatas a combinações eficazes de princípios ativos. O objetivo deste estudo foi o de encontrar alternativas de tratamentos alopáticos eficazes sobre uma população de nematódeos gastrintestinais resistente a todas as classes de medicamentos com ação nematicida disponíveis no mercado brasileiro, encontrada infectando naturalmente os ovinos do rebanho-alvo.

\section{MATERIAL E MÉTODOS}

Este estudo foi conduzido em um rebanho de aproximadamente 5000 ovinos da raça Merino Australiano, no estado do Rio Grande do Sul, na porção subtropical do Brasil, na região da Fronteira-Oeste, na divisa com o Uruguai. Conforme um estudo prévio realizado no mesmo rebanho, detectou-se resistência dos nematódeos gastrintestinais dos ovinos a todas as classes de fármacos com ação nematicida disponíveis para ruminantes no mercado brasileiro (Cezar et al. 2010b). Com base nesses resultados, foram selecionados aqueles fármacos com maior eficácia em relação à redução de ovos por grama de fezes (OPG) (Quadro 1) e/ou sobre um ou mais dos gêneros de nematódeos encontrados no rebanho (Quadro 2). Primou-se, além disso, por fármacos com amplo espectro de ação sobre nematódeos gastrintestinais. Dessa forma, elaboraram-se combinações de dois a dois princípios ativos e, adicionalmente, tratamentos com moxidectina no dobro da dose ou em alta concentração (longa ação) e com fosfato de levamisol no dobro da dose.

Quadro 1. Eficácias calculadas de drogas avaliadas no rebanho-alvo, em um Teste de Redução da Contagem de Ovos nas Fezes (FECRT), em um estudo prévio

\begin{tabular}{|c|c|c|c|}
\hline $\begin{array}{l}\text { Drogas testadas } \\
\text { em cada grupo } \\
\text { de ovinos }(n=15)\end{array}$ & $\begin{array}{c}\text { Dose } \\
\left(\mathrm{mg} / \mathrm{kg}^{-1}\right)\end{array}$ & $\begin{array}{c}\text { Eficácia } \\
\text { (FECRT) }\end{array}$ & $\begin{array}{c}\text { Nome comercial, } \\
\text { fabricante }\end{array}$ \\
\hline Fosfato de levamisol $22,3 \%$ & 4,5 & $23 \%$ & $\begin{array}{l}\text { Levamisol F, } \\
\text { Vetbrands }\end{array}$ \\
\hline Moxidectina $1 \%$ & 0,2 & $54 \%$ & $\begin{array}{l}\text { Cydectin ovinos, } \\
\text { Fort Dodge }\end{array}$ \\
\hline Sulfóxido de albendazol $10 \%$ & $\% \quad 2,5$ & $-2 \%$ & $\begin{array}{c}\text { Ricobendazole } 10, \\
\text { Ouro Fino }\end{array}$ \\
\hline Ivermectina $1 \%$ & 0,2 & $-42 \%$ । & Ivomec Injetável, Merial \\
\hline Nitrox & 9,7 & $32 \%$ & Dovenix Supra, Merial \\
\hline Disofenol $20 \%$ & 10,0 & $26 \%$ & Disofenol $20 \%$, IBASA \\
\hline $\begin{array}{l}\text { Triclorfon } 10 \% \\
\text { lvermectina }\end{array}$ & 100,0 & $-35 \%$ & Bevermex, IRFA \\
\hline Ivermectina & 0,2 & & \\
\hline $\begin{array}{l}\text { Levamisol } \\
\text { Albendazole }\end{array}$ & 7,5 & $68 \%$ & Trimix, Merial \\
\hline Albendazole & 5,0 & & \\
\hline Closantel $10 \%$ & 10,0 & $-23 \%$ & Diantel, IRFA \\
\hline
\end{tabular}


Quadro 2. Percentual de cada um dos três gêneros de nematódeos gastrintestinais - Haemonchus spp. (Haem.), Trichostrongylus spp. (Trich.), Ostertagia spp. (Ostert.) recuperados de culturas de larvas das fezes dos ovinos, antes (D0) e 12 dias depois (D12) dos tratamentos antiparasitários, em um estudo prévio (são apresentados apenas os resultados para as drogas selecionadas para 0 presente estudo)

\begin{tabular}{ccccccc}
\hline $\begin{array}{c}\text { Drogas testadas } \\
\text { em grupos de } \\
15 \text { ovinos }\end{array}$ & \multicolumn{2}{c}{ Haem. (\%) } & \multicolumn{2}{c}{ Trich. (\%) } & \multicolumn{2}{c}{ Ostert. (\%) } \\
\hline D12(PR ) & D0 & D12(PR) & D0 D12(PR) \\
\hline $\begin{array}{c}\text { Fosfato de } \\
\text { levamisol 22,3\% }\end{array}$ & 48 & $38(39) \mathrm{R}^{\mathrm{b}}$ & 52 & $56(17) \mathrm{R}$ & 0 & $6(0) \mathrm{R}$ \\
Moxidectina 1\% & 50 & $31(72) \mathrm{R}$ & 44 & $59(38) \mathrm{R}$ & 6 & $10(31) \mathrm{R}$ \\
Disofenol 20\% & 48 & $22(66) \mathrm{R}$ & 36 & $46(6) \mathrm{R}$ & 16 & $32(0) \mathrm{R}$ \\
Triclorfon 10\% & 42 & $36(0) \mathrm{R}$ & 52 & $41(0) \mathrm{R}$ & 6 & $23(0) \mathrm{R}$ \\
Closantel 10\% & 44 & $38(0) \mathrm{R}$ & 50 & $20(51) \mathrm{R}$ & 6 & $42(0) \mathrm{R}$ \\
\hline
\end{tabular}

a $P R=$ percentual de redução.

${ }^{\mathrm{b}} \mathrm{R}=$ resistente.

Foram utilizados oito grupos de 15 ovinos, formados aleatoriamente a partir de um lote de animais com cerca de 10 meses de idade, naturalmente infectados por nematódeos do trato gastrintestinal. Cada um dos tratamentos descritos no Quadro 3 foi testado sobre um desses oito grupos. Como regra, combinaram-se fármacos com mecanismos de ação diferentes. Não houve mistura física dos fármacos anteriormente às aplicações, as quais foram feitas por vias ou em pontos anatômicos distintos nos ovinos, seguindo-se as dosagens recomendadas nas bulas dos medicamentos (exceto nos grupos G1 e G2 em que se utilizaram doses mais altas propositalmente).

Coletaram-se amostras de fezes imediatamente antes dos tratamentos (D0) e doze dias após os mesmos (D12). A cada coleta, as amostras foram examinadas, conforme recomendações de Ueno \& Gonçalves (1998), por contagens individuais de OPG pela técnica de McMaster, com margem de detecção de 100 OPG, e por culturas de larvas, uma para cada grupo a cada coleta, pela técnica de Roberts \& O'Sullivan, com identificação de gênero de 100 larvas infectantes recuperadas de cada cultura.

A eficácia dos tratamentos testados foi calculada através de um Teste de Redução da Contagem de Ovos nas Fezes
(FECRT), pela fórmula: $P R=100$ (1-OPG final/OPG inicial), onde, "PR" é o percentual de redução de OPG; "OPG inicial" e "OPG final" são, respectivamente, a média aritmética dos valores de OPG para cada grupo imediatamente antes (D0), e doze dias depois (D12) dos tratamentos (Coles et al. 1992, Coles et al. 2006, McKenna 2006). Para ser considerado eficaz, o tratamento deve reduzir a contagem de OPG, e os percentuais de cada gênero de nematódeo nas culturas de larvas, em pelo menos $95 \%$, com limite inferior do intervalo de confiança a $95 \%$ maior do que $90 \%$. Esses cálculos foram realizados utilizando-se o RESO FECRT analysis software, versão 4.0 (disponível em: http://www.vetsci.usyd.edu.au/sheepwormcontrol, dentro do 'SiteMap').

\section{RESULTADOS E DISCUSSÃO}

As eficácias calculadas de cada um dos tratamentos testados encontram-se descritas no Quadro 4. Observou-se que apenas o tratamento composto por uma combinação de moxidectina 1\% (MOX 1\%) + disofenol 20\% (DISOF)

Quadro 4. Médias de OPG de cada grupo de ovinos, antes (D0) e 12 dias depois (D12) dos tratamentos

antiparasitários, e percentual de redução de OPG (PR) para cada tratamento no Teste de Redução da Contagem de Ovos nas Fezes (FECRT)

\begin{tabular}{|c|c|c|c|}
\hline \multirow{2}{*}{$\begin{array}{c}\text { Grupos }(n=15) \text { e } \\
\text { tratamentos testados }\end{array}$} & \multicolumn{2}{|c|}{ Média de OPG } & \multirow{2}{*}{$\begin{array}{l}\text { Resultado } \\
\text { (FECRT) }\end{array}$} \\
\hline & Do & $\mathrm{D} 12(\mathrm{PR})$ & \\
\hline $\begin{array}{l}\text { G1. Moxidectina 1\% } \\
\text { (dobro da dose) }\end{array}$ & 2900 & $313(89 \%)$ & Resistência \\
\hline $\begin{array}{l}\text { G2. Fosfato de levamisol } \\
22,3 \% \text { (dobro da dose) }\end{array}$ & 3971 & $682(83 \%)$ & Resistência \\
\hline $\begin{array}{l}\text { G3.Moxidectina } 1 \%+ \\
\text { fosfato de levamisol } 22,3 \%\end{array}$ & 4887 & $395(92 \%)$ & Resistência \\
\hline $\begin{array}{l}\text { G4.Moxidectina } 1 \%+ \\
\text { disofenol } 20 \%\end{array}$ & 3067 & $40(99 \%)$ & Eficácia \\
\hline $\begin{array}{l}\text { G5.Moxidectina 1\%+ } \\
\text { triclorfon } 10 \%\end{array}$ & 2538 & $238(91 \%)$ & Resistência \\
\hline $\begin{array}{l}\text { G6.Moxidectina 1\% + } \\
\text { closantel } 10 \%\end{array}$ & 5140 & $892(83 \%)$ & Resistência \\
\hline $\begin{array}{r}\text { G7.Fosfato de levamisol } \\
22,3 \%+\text { triclorfon } 10 \%\end{array}$ & 4227 & $1536(64 \%)$ & Resistência \\
\hline G8.Moxidectina $10 \%$ & 2773 & $598(78 \%)$ & Resistência \\
\hline
\end{tabular}

Quadro 3. Tratamentos avaliados no rebanho-alvo de ovinos no presente estudo

\begin{tabular}{|c|c|c|c|c|}
\hline & Grupos $(n=15)$ e tratamentos & $\begin{array}{c}\text { Dose } \\
\left(\mathrm{mg} / \mathrm{kg}^{-1}\right)^{\mathrm{a}}\end{array}$ & Classes & $\begin{array}{l}\text { Nome comercial, } \\
\text { fabricante }\end{array}$ \\
\hline G1. & Moxidectina 1\% (Dobro da dose) & 0,4 & Milbemicina ${ }^{b}$ & Cydectin ovinos, Fort Dodge \\
\hline G2. & Fosfato de levamisol 22,3\% (Dobro da dose) & 9,0 & Imidazotiazol & Levamisol F, Vetbrands \\
\hline \multirow[t]{2}{*}{ G3. } & Moxidectina $1 \%$ & 0,2 & Milbemicina ${ }^{b}$ & Cydectin ovinos, Fort Dodge \\
\hline & Fosfato de levamisol $22,3 \%$ & 4,5 & Imidazotiazol & Levamisol F, Vetbrands \\
\hline \multirow[t]{2}{*}{ G4. } & Moxidectina $1 \%$ & 0,2 & Milbemicina ${ }^{b}$ & Cydectin ovinos, Fort Dodge \\
\hline & Disofenol $20 \%$ & 10,0 & Nitrofenol & Disofenol $20 \%$, IBASA \\
\hline \multirow[t]{2}{*}{ G5. } & Moxidectina $1 \%$ & 0,2 & Milbemicinab & Cydectin ovinos, Fort Dodge \\
\hline & Triclorfon $10 \%$ & 100,0 & Organofosfato & Bevermex,IRFA \\
\hline \multirow[t]{2}{*}{ G6. } & Moxidectina $1 \%$ & 0,2 & Milbemicina ${ }^{b}$ & Cydectin ovinos, Fort Dodge \\
\hline & Closantel $10 \%$ & 10,0 & Salicilanilida & Diantel, IRFA \\
\hline \multirow[t]{2}{*}{ G7 } & Fosfato de levamisol $22,3 \%$ & 4,5 & Imidazotiazol & Levamisol F, Vetbrands \\
\hline & Triclorfon $10 \%$ & 100,0 & Organofosfato & Bevermex, IRFA \\
\hline G8. & Moxidectina $10 \%$ & 1,0 & Milbemicina ${ }^{b}$ & Onyx, Fort Dodge \\
\hline
\end{tabular}

a Todos por via parenteral, subcutânea, exceto o closantel 10\% (via oral).

b Lactona macrocíclica. 
Quadro 5. Percentual de cada um dos cinco gêneros de nematódeos gastrintestinais - Haemonchus spp. (Haem.), Trichostrongylus spp. (Trich.), Ostertagia spp. (Ostert.), Cooperia spp. (Coop.), Oesophagostomum spp. (Oesoph.) - recuperados de culturas de larvas em fezes ovinas coletadas antes (D0) e 12 dias depois (D12) dos tratamentos antiparasitários, e percentual de redução (PR), para cada gênero, calculado pelo RESO FECRT 4.0

\begin{tabular}{|c|c|c|c|c|c|c|c|c|c|c|}
\hline \multirow{2}{*}{$\begin{array}{l}\text { Grupos }(n=15) \\
\text { e tratamentos }\end{array}$} & \multicolumn{2}{|c|}{ Haem.(\%) } & \multicolumn{2}{|c|}{ Trich. (\%) } & \multicolumn{2}{|c|}{ Ostert. (\%) } & \multicolumn{2}{|c|}{ Coop. (\%) } & \multicolumn{2}{|c|}{ Oesoph. (\%) } \\
\hline & D0 & D12(PR) & Do & D12(PR) & Do & $\mathrm{D} 12(\mathrm{PR})$ & Do & D12(PR) & Do & D12(PR) \\
\hline $\begin{array}{l}\text { G1. Moxidectina } 1 \% \\
\text { (dobro da dose) }\end{array}$ & 78 & $52(93) r^{a}$ & 4 & $40(0) \mathrm{R}^{\mathrm{a}}$ & 0 & $2(0) R$ & 18 & $6(96) S^{a}$ & 0 & $O\left(N C^{b}\right)$ \\
\hline $\begin{array}{l}\text { G2. Fosf. lev. }{ }^{\mathrm{C}} 22,3 \% \\
\text { (dobro da dose) }\end{array}$ & 46 & $14(95) r$ & 16 & $80(14) R$ & 16 & $4(96) S$ & 20 & 2(98)S & 2 & $0(100) \mathrm{S}$ \\
\hline $\begin{array}{l}\text { G3. Moxidectina 1\% + } \\
\text { fosf. lev. } 22,3 \%\end{array}$ & 68 & $6(99) S$ & 4 & $91(0) R$ & 20 & $3(99) S$ & 8 & $0(100) S$ & 0 & $0(\mathrm{NC})$ \\
\hline $\begin{array}{l}\text { G4. Moxidectina } 1 \%+ \\
\text { disofenol } 20 \%\end{array}$ & 54 & $6(100) S$ & 10 & $86(89) R$ & 20 & $4(100) S$ & 16 & $4(100) S$ & 0 & $0(\mathrm{NC})$ \\
\hline $\begin{array}{l}\text { G5. Moxidectina } 1 \%+ \\
\text { triclorfon } 10 \%\end{array}$ & 80 & $26(97) S$ & 2 & $48(0) \mathrm{R}$ & 2 & $20(6) \mathrm{R}$ & 16 & $6(96) S$ & 0 & $0(\mathrm{NC})$ \\
\hline $\begin{array}{l}\text { G6. Moxidectina } 1 \%+ \\
\text { closantel } 10 \%\end{array}$ & 60 & $4(99) S$ & 24 & $86(38) R$ & 0 & $6(0) R$ & 12 & $0(100) S$ & 6 & $2(94) r$ \\
\hline $\begin{array}{l}\text { G7. Fosf. lev. } 22,3 \%+ \\
\text { triclorfon } 10 \%\end{array}$ & 62 & $8(95) \mathrm{S}$ & 8 & $62(0) \mathrm{R}$ & 4 & $18(0) \mathrm{R}$ & 26 & $8(89) \mathrm{R}$ & 0 & $4(0) \mathrm{R}$ \\
\hline G8. Moxidectina $10 \%$ & 58 & $47(83) R$ & 0 & $47(0) R$ & 2 & $6(35) \mathrm{R}$ & 40 & $0(100) S$ & 0 & $0(\mathrm{NC})$ \\
\hline
\end{tabular}

a $\mathrm{r}$ baixa resistência, $\mathrm{R}=$ resistente, $\mathrm{S}=$ susceptível.

b $\mathrm{NC}=$ não calculado.

c Fosf. lev. = fosfato de levamisol.

apresentou eficácia dentro do nível desejado (PR $\geq 95 \%$ ). Os tratamentos com MOX $1 \%$ (dobro da dose terapêutica recomendada), MOX 1\% + triclorfon 10\% (TRICLOR) e MOX $1 \%$ + fosfato de levamisol 22,3\% (LEVAM) apresentaram eficácia próxima aos $90 \%$. Todos os demais tratamentos foram insuficientes.

No Quadro 5 apresentam-se os gêneros de nematódeos gastrintestinais recuperados das culturas de larvas, e suas proporções antes (D0) e depois (D12) dos tratamentos. Detectaram-se maiores freqüências dos gêneros Haemonchus spp., Trichostrongylus spp., Ostertagia spp. e Cooperia spp., os quais apresentaram mais relevância em relação à resistência parasitária aos tratamentos em geral. O gênero Oesophagostomum spp. ocorreu com baixa prevalência, porém, detectou-se resistência deste gênero aos tratamentos a base de MOX 1\% + closantel $10 \%$ (CLOS) e de LEVAM + TRICLOR.

A combinação MOX 1\% + DISOF apresentou redução de OPG de 99\%, podendo, com isso, ser considerada altamente eficaz (Coles et al. 1992, Wood et al. 1995). Conforme se observa no Quadro 5, esse tratamento teve grande eficácia sobre Haemonchus spp., o que já era esperado, uma vez que ambas as drogas utilizadas nessa combinação haviam demonstrado, previamente, boa ação sobre este gênero (Quadro 2). Esse é um resultado de grande relevância, sabendo-se que a hemoncose é extremamente prejudicial à criação de ovinos, podendo ser considerada um dos principais problemas na ovinocultura ao redor do mundo, particularmente nas regiões tropicais e subtropicais (Echevarria et al. 1996, Cheah \& Rajamanickam 1997, Miller et al. 1998, Waller et al. 2004).

Além disso, levando-se em conta o baixo desempenho dos tratamentos convencionais testados anteriormente nes- te mesmo rebanho (Cezar et al. 2010b), pode-se considerar positiva a obtenção de resultados superiores aos $90 \%$ de eficácia (Wood et al. 1995, Sangster 1999), observados nos tratamentos: moxidectina $1 \%+$ triclorfon $10 \%$ (MOX $1 \%$ + TRICLOR), com PR=91\%, e moxidectina $1 \%$ + fosfato de levamisol 22,3\% (MOX 1\% + LEVAM), com PR=92\%, com a ressalva de que no primeiro caso observou-se alto grau de resistência dos gêneros Trichostrongylus spp. e Ostertagia spp. e, no segundo, do gênero Trichostrongylus spp. (Quadro 5). Dessa forma, tais combinações apenas seriam recomendáveis no caso de baixa incidência desses gêneros resistentes nos animais, ou com a utilização de um terceiro fármaco para o controle dos mesmos.

Por outro lado, as combinações de MOX 1\% + CLOS e de LEVAM + TRICLOR apresentaram eficácias insuficientes (Quadro 4). Deve-se notar que o CLOS foi incluído neste estudo devido à sua ação sobre Trichostrongylus spp., demonstrada anteriormente no rebanho-alvo (Quadro 2). Porém, este fármaco, além de não ser comumente indicado para o controle do referido gênero, havia apresentado, naquele estudo, $P R=-23 \%$, o que pode ter determinado o baixo desempenho de sua combinação com a MOX 1\%. De maneira semelhante, a combinação de dois fármacos com alto grau de resistência parasitária no rebanho-alvo (LEVAM + TRICLOR) teve baixíssima eficácia (Quadro 4). Esse fato pode estar, ainda, associado à ocorrência de mecanismos semelhantes de resistência parasitária (resistência cruzada) entre imidazotiazóis e organofosfatos (Sangster 1999). Isso demonstra que combinações aleatórias de drogas não são aconselháveis em hipótese alguma, sob pena de aumentarem os custos dos tratamentos sem acréscimos à eficácia dos mesmos, e podendo, inclusive, acentuar o problema de resistência parasitária (cruzada). 
As altas doses de MOX 1\% ou de LEVAM (ambas compatíveis ao dobro da dose terapêutica recomendada) não apresentaram eficácia satisfatória (Quadro 4). Tais tratamentos foram executados visando à obtenção de um aumento proporcional no pico de concentração de princípio ativo no plasma dos ovinos e, por conseguinte, nos tecidos-alvos, nos quais há contato do princípio ativo com os parasitas (Lanusse \& Prichard 1993, van Zeveren et al. 2007). Porém, tais subterfúgios não foram efetivos, assim como havia sido observado por Cezar et al. (2010a), em bovinos. Ademais, superdosagens tendem a intensificar a pressão de seleção de genótipos homozigotos resistentes (Smith et al. 1999) e os efeitos adversos dos tratamentos (Bakima et al. 1989, Borges et al. 1999), além de aumentarem seus custos por animal.

As lactonas macrocíclicas de longa ação, veiculadas em base oleosa, têm como característica um período estendido de absorção após a aplicação (Ranjan et al. 2010), caso da moxidectina $10 \%$ longa ação (MOX $10 \%$ LA). Isso significa que essas formulações têm a capacidade de prolongar o período de ação, mas não necessariamente de melhorar a eficácia do tratamento.

Ressaltando-se que a resistência parasitária à MOX $1 \%$ já havia sido demonstrada no rebanho-alvo em estudo prévio (Quadro 1), observou-se que a utilização da formulação de MOX 10\% LA não foi suficiente para o alcance da eficácia desejada ( $P R \geq 95 \%)$. Isso vai ao encontro de resultados anteriores que dão conta de que: o aumento da concentração de lactonas macrocíclicas, para as quais se tenha comprovado resistência parasitária, pode não resultar na recuperação da eficácia do tratamento (Cezar et al. 2010a). Essas observações indicam que a utilização de antiparasitários de longa ação (e com maior concentração de princípio ativo) deve ser recomendada apenas em casos em que se deseje ampliar o período de proteção para o rebanho. Em tempo, deve-se fazer a ressalva de que a formulação comercial de MOX 10\% LA disponível no mercado apenas possui indicação do fabricante para o uso em bovinos, tendo sido testada aqui, experimentalmente. No entanto, a moxidectina é um endectocida indicado e amplamente utilizado em ovinos (McKellar \& Jackson 2004).

A combinação de MOX 1\% + DISOF, além de ter resultado em maior redução de OPG em comparação aos demais tratamentos testados, superou a redução mínima de OPG esperada, ao alcançar um PR=99\%. Nesse caso, o fato de não se ter que recorrer a altas doses proporcionará uma menor pressão de seleção de genótipos homozigotos resistentes na população parasitária, e permitirá maior segurança no que diz respeito ao risco de efeitos adversos exceto possíveis efeitos devidos à interação medicamentosa, os quais precisam ser avaliados. Além disso, ao se evitarem os fármacos de alta concentração (com longa ação), também se evita acelerar o processo de resistência parasitária, uma vez que, quando utilizados, estes proporcionam longo período de sub-dose residual (Barnes et al. 1995, Dobson et al. 1996, Yazwinski et al. 2006), prejudicando a refugia, a qual, provavelmente, já se encontra bastante comprometida no caso em questão (Coles 2002, van Wyk et al. 2006). Por sua vez, quando utilizadas nas suas doses terapêuticas recomendadas, e nas suas formulações de menor concentração, os anti-helmínticos facilitam um manejo adequado à manutenção da refugia nas pastagens (Molento 2009), o que é essencial para a sustentabilidade dos tratamentos.

Outra questão a ser considerada é que a combinação de fármacos tenderá a ser mais efetiva quando forem utilizados princípios ativos com mecanismos de ação diferentes. Isto porque, dessa forma é maior a probabilidade de que se somem os efeitos de ambos, uma vez que serão necessários diferentes mecanismos adaptativos dos parasitas para que estes resistam à combinação de princípios ativos, e isso tenderá a dificultar o desenvolvimento da resistência parasitária (Hall et al. 1981, Barnes et al. 1995).

O gênero Trichostrongylus spp. foi resistente, e aumentou sua proporção, após todos os tratamentos (Quadro 5). Esse gênero foi o principal limitante para a eficácia das combinações de drogas utilizadas. Por conta disso, devem-se buscar alternativas para um controle adequado de Trichostrongylus spp. no rebanho-alvo deste estudo. Contudo, considerando-se a situação extrema da resistência parasitária encontrada acometendo o mesmo, pode-se classificar a resistência do gênero Trichostrongylus spp. como aceitável, desde que a eficácia do tratamento supere os $95 \%$. Ainda, conforme se observa no Quadro 5, recuperaram-se larvas resistentes dos gêneros Haemonchus spp. e Ostertagia spp. após todos os tratamentos (embora isso não configure, necessariamente, uma resistência genérica considerável). Da mesma forma, larvas dos gêneros Cooperia spp. e Oesophagostomum spp. resistiram a alguns dos tratamentos.

Percebe-se, com base nos resultados deste estudo, que as combinações de fármacos poderão apresentar eficácias mais ou menos satisfatórias dependendo do(s) gênero(s) de nematódeo(s) predominante(s) no rebanho. Deve-se considerar que tanto a própria eficácia dos tratamentos, quanto os fatores epidemiológicos locais, terão influência na carga parasitária e na proporção de cada gênero na população de parasitas em um rebanho (Barger 1999, Molento 2009). Assim, pesa contra os antiparasitários a base de combinações de princípios ativos disponíveis no mercado, o fato de que estes não possibilitam que a escolha das bases químicas a serem aplicadas seja feita conforme as características da população parasitária em cada caso, especificamente.

Embora haja relatos de resistência lateral de endoparasitas à moxidectina em rebanhos tratados com outras lactonas macrocíclicas (Vermunt et al. 1996), aparentemente, a resistência à moxidectina se desenvolve mais lentamente do que a resistência às avermectinas (Ranjan et al. 2002, Cezar et al. 2010a). Isso torna a moxidectina uma boa candidata para associação com outras drogas em casos de resistência múltipla. De qualquer forma, as combinações de fármacos a serem utilizadas sobre populações parasitárias multirresistentes devem ser baseadas 
em testes de eficácia dessas drogas, incluindo culturas de larvas, em cada rebanho. Além disso, sua eficácia deve ser mantida sob monitoramento.

\section{CONCLUSÕES}

Demonstrou-se a viabilidade da recuperação da eficácia dos tratamentos anti-helmínticos em um rebanho ovino acometido por nematódeos resistentes a todas as classes de drogas com ação nematicida disponíveis no mercado.

A adequada combinação de drogas antiparasitárias, com diferentes mecanismos de ação, pode proporcionar alta eficácia no controle de uma população de nematódeos gastrintestinais resistente (inclusive às drogas utilizadas nas combinações), e sem implicar na necessidade de maiores doses ou concentrações dos princípios ativos.

\section{REFERÊNCIAS}

Albonico M., Bickle Q., Ramsan M., Montresor A., Savioli L. \& Taylor M. 2003. Efficacy of mebendazole and levamisole alone or in combination against intestinal nematode infections after repeated targeted mebendazole treatment in Zanzibar. Bull. World Health Organ. 81:343-352.

Anderson N., Martin P.J. \& Jarret R.G. 1988. Mixtures of anthelmintics: A strategy against resistance. Aust. Vet. J. 65:62-64.

Bakima M., Baudet H.M., Lekeux P. \& Lomba F. 1989. Respiratory and pulmonary haemodynamic changes during experimental organophosphate poisoning in goats. Vet. Res. Comm. 13:127-133.

Barger I.A. 1999. The role of epidemiological knowledge and grazing management for helminth control in small ruminants. Int. J. Parasitol. 29:41-47.

Barnes E.H., Dobson R.J. \& Barger I.A. 1995. Worm control and anthelmintic resistance: Adventures with a model. Parasitol. Today 11:56-63.

Borges A.S., Mendes L.C., de Andrade A.L., Machado G.F. \& Peiro J.R. 1999. Optic neuropathy in sheep associated with overdosage of closantel. Vet. Hum. Toxicol. 41:378-380.

Cezar A.S., Vogel F.S.F., Sangioni L.A., Antonello A.M., Camillo G., Toscan G. \& Araujo L.O. 2010a. Ação anti-helmíntica de diferentes formulações de lactonas macrocíclicas em cepas resistentes de nematódeos de bovinos. Pesq. Vet. Bras. 30:523-528.

Cezar A.S., Toscan G., Camillo G., Sangioni L.A., Ribas H.O. \& Vogel F.S.F. 2010b. Multiple resistance of gastrointestinal nematodes to nine different drugs in a sheep flock in southern Brazil. Vet. Parasitol. 173:157-160.

Cheah T.S. \& Rajamanickam C. 1997. Epidemiology of gastro-intestinal nematodes of sheep in wet tropical conditions in Malaysia. Trop. Anim. Health Prod. 29:165-173.

Coles G.C. 2002. Sustainable use of anthelmintics in grazing animals. Vet. Rec. 151:165-169.

Coles G.C., Bauer C., Borgsteede F.H.M., Geerts S., Klei T.R., Taylor M.A. \& Waller P.J. 1992. World Association for the Advancement of Veterinary Parasitology (WAAVP) methods for the detection of anthelmintic resistance in nematodes of veterinary importance. Vet. Parasitol. 44:35-44.

Coles G.C., Jackson F., Pomroy W.E., Prichard R.K., von SamsonHimmelstjerna G., Silvestre A., Taylor M.A. \& Vercruysse J. 2006. The detection of anthelmintic resistance in nematodes of veterinary importance. Vet. Parasitol. 136:167-185.

Dobson R.J., LeJambre L. \& Gill J. 1996. Management of anthelmintic resistance: inheritance of resistance and selection with persistent drugs. Int. J. Parasitol. 26:993-1000.
Echevarria F., Borba M.F.S., Pinheiro A.C., Waller P.J. \& Hansen J.W. 1996. The prevalence of anthelmintic resistance in nematode parasites of sheep in Southern Latin America: Brazil. Vet. Parasitol. 62:199-206.

Entrocasso C., Alvarez L., Manazza J., Lifschitz A., Borda B., Virkel G., Mottier L. \& Lanusse C. 2008. Clinical efficacy assessment of the albendazole-ivermectin combination in lambs parasitized with resistant nematodes. Vet. Parasitol. 155:249-256.

Furlong J., Prata M.C.A. \& Martins J.R. 2007. O carrapato dos bovinos e a resistência: temos o que comemorar? Hora Vet. 159:26-32.

Geerts S. \& Gryseels B. 2000. Drug resistance in human helminths: current situation and lessons from livestock. Clin. Microbiol. Rev. 13:207-222.

George J.E., Pound J.M. \& Davey R.B. 2004. Chemical control of ticks on cattle and the resistance of these parasites to acaricides. Parasitol. 129:353-366.

Hall C.A., Kelly J.D., Whitlock H.V. \& Ritchie L. 1981. Prolonged anthelmintic effect of closantel and disophenol against a thiabendazole selected resistant strain of Haemonchus contortus in sheep. Res. Vet. Sci. 31:104-106.

Hopkins A.L., Witty M.J. \& Nwaka S. 2007. Mission possible. Nature 449:166-169.

Hu Y., Platzer E.G., Bellier A. \& Aroian R.V. 2010. Discovery of a highly synergistic anthelmintic combination that shows mutual hypersusceptibility. PNAS 107:5955-5960. Disponível em <http:// www.pnas.org/cgi/doi/10.1073/pnas.0912327107> Acesso em 5th mar. 2010.

Lanusse C.E. \& Prichard R.K. 1993. Relationship between pharmacological properties and clinical efficacy of ruminant anthelmintics. Vet. Parasitol. 49:123-158.

Martins J.R., Furlong J., Prata M.C.A. \& Doyle R.L. 2008. Acaricide resistance in Brazil and the use of mixtures as chemical alternative for tick control. VI Seminario Internacional de Parasitología Animal, Boca del Río Veracruz, Mexico, 4p. Disponível em <http://www.cbpv.com.br/ artigos/CBPV_artigo_026.pdf> Acesso em 13.09. 2010.

McKellar Q.A. \& Jackson F. 2004. Veterinary anthelmintics: Old and new. Trends Parasitol. 20:456-461.

McKenna P.B. 2006. Further comparison of faecal egg count reduction test procedures: sensitivity and specificity. N. Z. Vet. J. 54:365-366.

Miller D.K. \& Craig T.M. 1996. Use of anthelmintic combinations against multiple resistant Haemonchus contortus in Angora goats. Small Rumin. Res. 19:281-283.

Miller J.E., Bahirathan M., Lemarie S.L., Hembry F.G., Kearney M.T. \& Barras S.R. 1998. Epidemiology of gastrointestinal nematode parasitism in Suffolk and Gulf Coast Native sheep with special emphasis on relative susceptibility to Haemonchus contortus infection. Vet. Parasitol. 74:55-74.

Molento M.B. 2009. Parasite control in the age of drug resistance and changing agricultural practices. Vet. Parasitol. 163:229-234.

Mwamachi D.M., Audho J.O., Thorpe W. \& Baker R.L. 1995. Evidence for multiple anthelmintic resistance in sheep and goats reared under the same management in coastal Kenya. Vet. Parasitol. 60:303-313.

Pink R., Hudson A., Mouriès M.-A. \& Bendig M. 2005. Opportunities and challenges in antiparasitic drug discovery. Nature Rev. Drug Discov. 4:727-739.

Ranjan S., Search R., Szewczyk E., Amodie D., Pollet R. \& Rugg D. 2010. Evaluation of the persistent activity of moxidectin (10\%) longacting (LA) injectable formulation against Dictyocaulus viviparus, Haemonchus placei, Trichostrongylus axei and Oesophagostomum radiatum infections in cattle. Vet. Parasitol. 167:50-54.

Ranjan S., Wang G.T., Hirschlein C. \& Simkins K.L. 2002. Selection for resistance to macrocyclic lactones by Haemonchus contortus in sheep. Vet. Parasitol. 103:109-117. 
Sangster N.C. 1999. Anthelmintic resistance: Past, present and future. Int. J. Parasitol. 29:115-124.

Sargison N.D., Jackson F., Bartley D.J., Wilson D.J., Stenhouse L.J. \& Penny C.D. 2007. Observations on the emergence of multiple anthelmintic resistance in sheep flocks in the south-east of Scotland. Vet. Parasitol. 145:65-76.

Smith G., Grenfell B.T., Isham V. \& Cornell S. 1999. Anthelmintic resistance revisited: Under-dosing, chemoprophylactic strategies, and mating probabilities. Int. J. Parasitol. 29:77-91.

Traversa D., Paoletti B., Otranto D. \& Miller J. 2007. First report of multiple drug resistance in trichostrongyles affecting sheep under field conditions in Italy. Parasitol. Res. 101:1713-1716.

Ueno H. \& Gonçalves V.C. 1998. Manual para diagnóstico das helmintoses de ruminantes. Tóquio: Japan International Cooperation Agency. 143p.

van Wyk J.A., Hoste H., Kaplan R.M. \& Besier R.B. 2006. Targeted selective treatment for worm management: How do we sell rational programs to farmers? Vet. Parasitol. 139:336-346.

van Zeveren A.M., Casaert S., Alvinerie M., Geldhof P., Claerebout E. \& Vercruysse J. 2007. Experimental selection for ivermectin resistance in Ostertagia ostertagi in cattle. Vet. Parasitol. 150:104-110.
Vermunt J., West D. \& Pomroy W. 1996. Inefficacy of moxidectin and doramectin against ivermectin-resistant Cooperia spp. of cattle in New Zealand. N. Z. Vet. J. 44:188-193.

Waller P.J. \& Thamsborg S.M. 2004. Nematode control in 'green' ruminant production systems. Trends Parasitol. 20:493-497.

Waller P.J., Rudby-Martin L., Ljungström B.L. \& Rydzik A. 2004. The epidemiology of abomasal nematodes of sheep in Sweden, with particular reference to over-winter survival strategies. Vet. Parasitol. 122:207-220

Wood I.B., Amaral N.K., Bairden K., Duncan J.L., Kassai T., Malone Jr J.B., Pankavich J.A., Reinecke R.K., Slocombe O., Taylor S.M. \& Vercruysse J. 1995. World Association for the Advancement of Veterinary Parasitology (W.A.A.V.P.) second edition of guidelines for evaluating the efficacy of anthelmintics in ruminants (bovine, ovine, caprine). Vet. Parasitol. 58:181-213.

Woods D.J. \& Williams T.M. 2007. The challenges of developing novel antiparasitic drugs. Invert. Neurosci. 7:245-250.

Yazwinski T.A., Williams J.C., Smith L.L., Tucker C., Loyacano A.F., DeRosa A., Peterson P., Bruer D.J. \& Delay R.L. 2006. Dose determination of the persistent activity of moxidectin long-acting injectable formulations against various nematode species in cattle. Vet. Parasitol. 137:273-285. 